\title{
DESENVOLVIMENTO DE CABINE DE SUBESTAÇÃO EM MÉDIA TENSÃO ${ }^{1}$
}

\author{
Gabriel Santiago Raimundo Rodrigues ${ }^{2}$ \\ Gustavo Lobato Campos ${ }^{3}$ \\ Letivan Cambraia Freire Junior ${ }^{4}$ \\ Lucas Alves de Oliveira ${ }^{5}$
}

\begin{abstract}
RESUMO
A energia elétrica, nos dias de hoje, é indispensável para a vida humana devido à contínua evolução tecnológica, perceptível, por exemplo: com o aumento da automação industrial, interligação de redes de conhecimento, dentre outros. As unidades industriais e grandes aglomerados comerciais demandam grande proporção da energia produzida em nosso país, e desta forma, optam, muitas vezes, pela compra de energia elétrica em média/alta tensão. Escolha válida pelo seu baixo custo quando comparada ao fornecimento em baixa tensão. Sendo assim, o objetivo deste trabalho é apresentar um artigo relacionado ao desenvolvimento de uma cabine de subestação em média tensão, especificamente a tipo 2: Medição, proteção com ou sem transformação (CEMIG, 2013), na Fábrica de Refrigerantes Mantiqueira localizada na cidade de Itamonte-MG. O desenvolvimento deste trabalho teve por base as literaturas da área mais especificamente as normativas das concessionárias de energia, especialmente as da Companhia Energética de Minas Gerais (CEMIG), destaca-se a norma ND 5.3 - Fornecimento de Energia Elétrica em Média Tensão, norma esta consoante com atos e normativas da Agência Nacional de Energia Elétrica (ANEEL) e da Associação Brasileira de Normas Técnicas (ABNT). Neste trabalho apresenta-se a escolha da entrada de energia da subestação, o levantamento de carga do consumidor, o diagrama unifilar e os procedimentos e execução dos setores de medição, proteção e transformação da subestação. A referida obra foi aprovada recebendo elogios da concessionária e está em pleno funcionamento.
\end{abstract}

Palavras-chave: Fornecimento de Energia Elétrica. Subestação. Média Tensão. CEMIG.

\section{INTRODUÇÃO}

O Sistema Elétrico de Potência é composto pelos sistemas de geração, transmissão e distribuição. Segundo Banco de Informação de Geração, (BIG), da Agência Nacional de

\footnotetext{
${ }^{1}$ Como citar este artigo:

RODRIGUES, G. S. R. et al. Desenvolvimento de cabine de subestação em média tensão. ForScience: revista científica do IFMG, Formiga, v. 5, n. 1, e00248, jan./jun. 2017.

${ }^{2}$ Graduado em Engenharia Elétrica e Proprietário da empresa ENGGIMAX. E-mail: gabriel.enggimax@gmail.com.

3 Doutorando em Engenharia Nuclear pela Universidade Federal de Minas Gerais (UFMG). Professor do Instituto Federal Minas Gerais - Campus Formiga. (http://lattes.cnpq.br/9613750934178733). E-mail: gustavo.lobato@ifmg.edu.br.

${ }^{4}$ Graduando em Engenharia Elétrica e Proprietário da Empresa Kz Engenharia. (http://lattes.cnpq.br/9668623706878073).E-mail: letivanjr@gmail.com.

${ }^{5}$ Graduando em Engenharia Elétrica. E-mail: lucasalves_arcos@ hotmail.com.
} 
Energia Elétrica (ANEEL), na data de 24/03/2017, o Brasil possui cerca de 151,6 GW de geração de potência instalada, excluindo a quantidade de energia comprada dos países vizinhos (ANEEL, 2017). Todo sistema de geração é conectado às fontes de consumo pelos segmentos de transporte (transmissão e distribuição).

O ramo industrial brasileiro e aglomerados comerciais demandam cerca de $57 \%$ da energia elétrica do país (EPE, 2014). Com o aumento do preço da energia, devido à seca dos últimos anos, e eventual expansão industrial ou comercial, buscando reduzir gastos, estes consumidores de energia elétrica têm optado pela compra de energia em tensões mais elevadas, média ou alta tensão.

Porém, para ser optante deste modelo de compra e fornecimento o consumidor deve atender as normas técnicas da concessionária de energia a que está interligado e possuir um valor mínimo de demanda estipulado pela mesma. Em Minas Gerais, a regulamentação é feita pela concessionária de energia Companhia Energética de Minas Gerais, (CEMIG). Embora o consumidor esteja conectado ao segmento de transporte de energia elétrica pertencente a CEMIG®, este não é obrigado a comprar energia diretamente da concessionária e poderá optar pelo mercado livre de energia, comércio este possível devido à interligação de todo sistema elétrico brasileiro. Neste caso, o consumidor paga tarifas referentes ao transporte da energia feita pela concessionária e deve respeitar às normas vigentes da mesma.

A CEMIG® em seu acervo técnico possui normas de distribuição sendo a ND - 5.3 Fornecimento de Energia Elétrica em Média Tensão Rede de Distribuição Aérea ou Subterrânea, a norma vigente para o fornecimento de energia nestes moldes. Esta norma é consonante com todas as normas de seu acervo técnico: com as normas da Associação Brasileira de Normas Técnicas (ABNT), NBR - 5410 (Instalações Elétricas de Baixa tensão), NBR 14039 (Instalações Elétricas de Média Tensão); com as Resoluções 395/2009, 414/2010 e 479/2012 da ANEEL, assim como as últimas resoluções, atos ou normativas do Conselho Regional de Engenharia e Agronomia de Minas Gerais, CREA-MG.

A norma ND 5.3 expõe critérios para o fornecimento de energia elétrica trifásica em média tensão, com valores nominais de $13,8 \mathrm{kV}, 22 \mathrm{kV}$ e $34,5 \mathrm{kV}$, para demandas superiores a $75 \mathrm{~kW}$ independentemente da localização ou atividade econômica. Dentre os parâmetros expostos, há a necessidade da construção de uma Subestação de Energia Elétrica, tema que será apresentado neste trabalho.

Subestação de Energia Elétrica é o ponto de ligação entre a rede de transmissão/distribuição da concessionária de energia e o consumidor (BARROS; GREDA, 2013). Sendo assim, fica definida, pela CEMIG®, como subestação a instalação 
compreendendo o ramal de entrada, poste ou pontalete particular, caixas, dispositivo de proteção, aterramento e ferragens, de responsabilidade do consumidor, preparada de forma a permitir a ligação da unidade consumidora a rede de transporte da concessionária (CEMIG, 2013).

Neste artigo, será apresentada uma situação de compra de energia elétrica em média tensão pertencente ao ramo industrial do estado de Minas Gerais, empregando como solução técnica a construção de uma subestação. Os critérios de dimensionamento e parametrização dos materiais, ferramentas e equipamentos dos setores de medição e proteção de uma Subestação de Energia Elétrica serão apresentados e discutidos.

O estudo de caso apresentado refere-se a Fábrica de Refrigerantes Mantiqueira®, localizada no município de Itamonte/MG. Devido a expansão do mercado de refrigerantes, a demanda contratada foi de 400kVA com previsão de aumento para $1 \mathrm{MVA}$, com tensão de fornecimento de $13,8 \mathrm{kV}$. O tipo de subestação nomeada em norma da CEMIG® foi a subestação n 2 - Medição, Proteção com ou sem Transformação. O transformador instalado dentro da subestação tem potência nominal de 1MVA a óleo isolante, possuindo tensão primária de $13,8 \mathrm{kV}$ e secundária de $127 / 220 \mathrm{~V}$, para alimentar toda fábrica.

\section{REFERENCIAL TEÓRICO}

\subsection{Sistema Elétrico de Potência}

O Sistema Elétrico de Potência, SEP, é constituído pelos seguimentos de geração, transmissão e distribuição de energia elétrica, sendo ele responsável por transportar fluxos de potência às cargas (consumidores) a ele ligadas (KAGAN; OLIVEIRA; ROBBA, 2005). Esta potência é quantificada pela tensão de fornecimento multiplicada pela corrente necessária para suprir a demanda de consumo conforme Equação 1 (BARROS; GEDRA, 2013).

$$
S=V \times I
$$

Onde: $S$ é o valor nominal de potência total transmitido, em volt-ampere[VA];

$V$ é o valor nominal de tensão da rede ou sistema, em volt[ $[V]$;

$I$ é o valor nominal de corrente transmitida pelo sistema, em ampere[A]. 
A potência $S$ entregue às cargas possui valor fixo, com os valores de tensão $V$ e corrente $I$ variáveis. Buscando reduzir custos e perdas de energia ao longo da transmissão, opta-se por reduzir os valores de corrente e elevar os valores de tensão transmitidos. Tal ação é justificada, pois, quanto maiores os valores de corrente maior será a bitola do condutor de energia. Além disto, uma ampliação na bitola do condutor acarreta um maior peso do material promovendo um maior esforço das torres de transmissão, sendo necessário reforço nas mesmas (BARROS; GEDRA, 2013).

Toda esta economia é repassada aos consumidores através de valores de tarifas de energia mais baixos. Porém, os níveis de tensão elevados possuem classificação conforme seus valores. Segundo a NR10 - Norma Regulamentadora $\mathrm{n}^{\circ} 10$ do Ministério do Trabalho e Emprego, todo valor de tensão superior a $1 \mathrm{kV}$ é considerado alta tensão. Segundo a ABNT, as tensões de valores inferiores a $1 \mathrm{kV}$ são consideradas baixa tensão; as tensões com valores compreendidos entre $1 \mathrm{kV}$ e $36,2 \mathrm{kV}$ são denominadas média tensão, valores superiores a $36,2 \mathrm{kV}$ são considerados alta tensão. Assim, é importante destacar, que este trabalho seguirá a nomenclatura da ABNT.

\subsection{Classificação dos Consumidores}

Segundo a ANEEL (2010), em sua resolução 414 de 09/09/2010, os consumidores de energia elétrica são classificados conforme o valor de tensão por eles recebido. Os consumidores são subdivididos entre os que recebem energia em baixa tensão (Grupo B) e os que recebem energia em alta tensão (Grupo A). O grupo de consumidores de energia de alta tensão são novamente divididos, em seis grupos, conforme destacado na Tabela 1.

\begin{tabular}{cc}
\multicolumn{2}{c}{ Tabela 1 - Classificação dos Consumidores de Energia em Alta Tensão } \\
\hline Grupo de Consumidor & Tensão de Fornecimento \\
\hline A1 & Igual ou superior a $230 \mathrm{kV}$ \\
A2 & Entre $88 \mathrm{kV}$ e $138 \mathrm{kV}$ \\
A3 & De $69 \mathrm{kV}$ \\
A3a & Entre $30 \mathrm{kV}$ e $44 \mathrm{kV}$ \\
A4 & Entre $2,3 \mathrm{kV}$ e $25 \mathrm{kV}$ \\
AS & Subterrânea inferior a $2,3 \mathrm{kV}$ \\
\hline
\end{tabular}

Fonte: Barros; Gedra, (2013). 
Para a tarifação do consumo de energia elétrica leva-se em conta a qual grupo de consumidor o cliente pertence, quanto maior o valor de tensão por ele recebido menor a tarifação pelo consumo de energia. Além disto, o consumidor pode optar por três tipos de tarifação: convencional, horo sazonal verde e horo sazonal azul; distintos entre si pelos valores cobrados em período de ponta, fora de ponta, para períodos úmidos e secos e os reflexos de variação dos níveis de tensão durante o dia. Os valores e os tipos de tarifas variam conforme a concessionária de energia a qual o cliente está conectado.

\subsection{Adequações do consumidor para recebimento de energia}

Para o consumidor receber energia, em média/alta tensão, o mesmo deve respeitar alguns requisitos. Segundo a ANEEL (2010), em sua resolução 414, caso o consumidor possua demanda entre $75 \mathrm{~kW}$ e $2500 \mathrm{~kW}$ o mesmo deve receber energia elétrica em tensão inferior a $69 \mathrm{kV}$. Em caso de demanda superior a $2500 \mathrm{~kW}$ o consumidor deve receber energia elétrica em tensão igual ou superior a 69kV (ANEEL, 2010).

O consumidor que optar pelo recebimento de energia elétrica em média/alta tensão deve arcar com os custos da infraestrutura de recebimento e transformação dessa energia. É necessária, então, a construção de uma subestação. Uma subestação é o ponto de conexão, entre o consumidor e a concessionária de energia, onde se recebe a energia em média/alta tensão, composto por equipamentos e componentes responsáveis pela medição, proteção e transformação de energia (CEMIG, 2013).

São documentados vários tipos e configurações de subestação, e neste trabalho será apresentada apenas a Subestação $\mathrm{n}^{\circ} 2$ exposta na norma ND 5.3 da CEMIG®. Mais especificamente com tensão de fornecimento fase-fase trifásico em delta de $13,8 \mathrm{kV}$ com frequência de $60 \mathrm{~Hz}$.

A Subestação $\mathrm{n}^{\circ}$ 2: Medição e proteção com ou sem transformação é um cubículo em alvenaria, usado para qualquer valor de demanda superior a $75 \mathrm{~kW}$, ilustrado na Figura 1. A medição de energia consumida é feita a 3 elementos de fase em média tensão. A proteção é feita por disjuntores e relés de proteção em média tensão. A transformação, se houver, acontecerá logo após o cubículo de proteção. Se não houver transformação em seu interior, cabos de média/alta tensão (três cabos fase e um cabo neutro) saem da subestação até o transformador(es) (BARROS; GEDRA, 2013). 


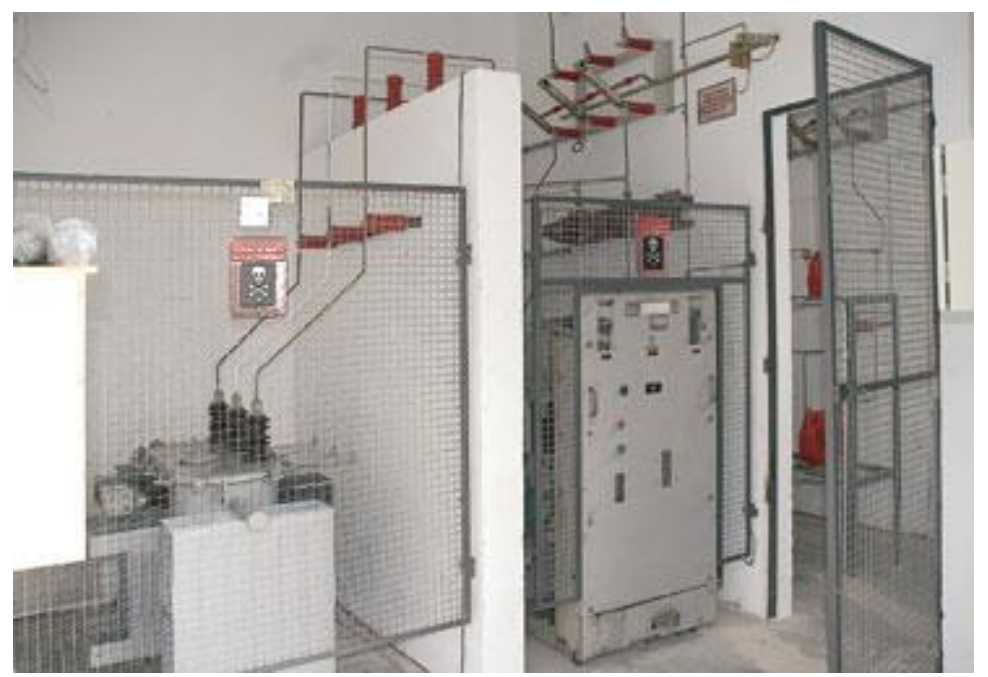

Figura 1 - Subestação Tipo 2 - Medição, Proteção com ou sem Transformação Fonte: Silva; Carvalho (2016)

Para ligação de uma subestação de energia elétrica existem processos burocráticos que devem ser cumpridos. Cada concessionária de energia possui seu procedimento de ligação, porém, é possível descrever algumas generalidades. Primeiramente, antes de qualquer processo de construção, o consumidor deve pedir a concessionária um estudo de viabilidade da construção de uma subestação, neste estudo o consumidor terá ciência se a rede a qual ele irá se interligar terá ou não condições de suportar a carga demandada por ele. Em caso negativo, a concessionária deverá fazer a adequação de rede, que poderá gerar custos ao consumidor (BARROS; GEDRA, 2013). Quando a concessionária escolhida é a CEMIG®, constatada a necessidade de adequação de rede, o consumidor arca com os custos e doa à concessionária toda a construção, recebendo pela doação créditos futuros em faturas posteriores (CEMIG, 2013). Existem regras que definem a participação financeira do consumidor e estão estabelecidas na Resolução 250 de 2007 da ANEEL. Porém se o consumidor não aceitar arcar com estes custos o processo está encerrado.

Caso haja viabilidade ou, assumida a responsabilidade pelo consumidor em arcar os custos, firma-se um contrato entre a concessionária de energia e o consumidor. A partir de então, o consumidor elabora o projeto de subestação e apresenta à concessionária de energia. O projeto deve ser confeccionado por um responsável técnico capacitado, habilitado e registrado no CREA, órgão que expedirá uma Anotação de Responsabilidade Técnica (ART), que será anexada ao projeto. O projeto pode ser aprovado diretamente, ou reprovado para que sejam feitas as adequações necessárias. Com o projeto definitivamente aprovado, o consumidor deve obter os materiais e equipamentos necessários para a construção da subestação (BARROS; GEDRA, 2013). 
Após a construção da subestação o cliente faz um pedido de inspeção à concessionária. Em caso de aprovação o consumidor solicita a ligação e a subestação é finalmente ligada. Havendo a reprovação, o consumidor deverá providenciar as adequações necessárias e pedir nova vistoria.

Embora conectado à rede da concessionária de energia, o consumidor não é obrigado a comprar energia direto da concessionária, pagando apenas os gastos de transmissão. Para optar por este tipo de compra o consumidor deve entrar no mercado de energia livre.

$\mathrm{O}$ mercado livre de energia foi instaurado pelo então presidente Fernando Henrique Cardoso em 1995 visando criar maior concorrência e competitividade entre as empresas brasileiras sendo controlado pela ANEEL. Neste mercado o consumidor de energia elétrica poderá escolher seu fornecedor por toda extensão do Sistema Interligado Nacional (SIN) (CELESC, 2016). Embora o consumidor seja optante do mercado de energia livre o mesmo deve se adequar as normas da concessionária de energia a ele ligada.

A inserção do consumidor ao mercado de energia livre deve ocorrer por meio de participações de pregões e o consumidor não pode ter contrato vigente com nenhuma concessionária. O consumidor pode participar do pregão pessoalmente ou por intermédio de agentes de comercialização. A ANEEL possui legislação, atos e resoluções referentes ao mercado de energia livre que devem ser seguidos pelos fornecedores, consumidores e agentes de comercialização (COMERC, 2016).

\section{MATERIAIS E MÉTODOS}

Toda subestação é composta basicamente por: ramal de entrada, setor de medição, setor de proteção e setor de transformação. Neste capítulo falaremos sobre cada um dos seguimentos.

\subsection{Ramal de Entrada}

Ramal de entrada é o ponto de ligação entre a rede da concessionária de energia e o ponto de medição de consumo de energia elétrica do consumidor. A conexão dos cabos de fase é feita aos barramentos de fase da subestação, os condutores são unipolares, de alumínio, isolados com XLPE- $90^{\circ} \mathrm{C}$ ou EPR- $90^{\circ} \mathrm{C}$, ligados conforme tensão de distribuição. Os condutores podem também ser de cobre, neste caso, são ligados diretamente ao aterramento da subestação. 
O ramal de entrada pode ligado de maneira aérea, desde que não corte terreno de terceiros, ou de maneira subterrânea, com os condutores passando por dutos e pela caixa de inspeção da concessionária. Em Minas Gerais, a norma usada para recebimento de energia aérea é a Norma da CEMIG ND 5.2, que estabelece parâmetros para este tipo de recebimento. Já para entrada de energia subterrânea, usa-se a Norma da CEMIG ND 2.3.

\subsection{Medição de Energia Consumida}

O setor de medição de energia elétrica é o setor de responsabilidade da concessionária de energia, que gera a fatura de conta de energia. Os equipamentos de medição, Transformadores de Corrente (TCs) e Transformadores de Potencial (TPs) de sinal, registradores eletrônicos e chaves de aferição somente serão ligados e instalados após a vistoria da concessionária (CEMIG, 2013). O dimensionamento desses materiais é determinado por normas das concessionárias. A Norma ND 5.3 da CEMIG contém o dimensionamento dos materiais usados para as tensões de fornecimento de sua rede, além de exemplificar a mesa suporte para os TCs e TPs de sinal de medição.

\subsection{Equipamentos de proteção}

Proteção de subestação são os equipamentos responsáveis por seccionamento do circuito de alimentação, ou eliminação de sobrecarga em caso de interferências, causadas por sobre-tensão, sobre-corrente, sub-tensão, harmônicos, etc., que podem causar danos materiais e físicos e/ou óbito as pessoas próximas. Os equipamentos usados, geralmente, são: relé microprocessado, TC's e TPs de sinal de proteção, disjuntor, chave seccionadora com fusível, aterramento (MAMEDE FILHO; MAMEDE, 2011).

Os disjuntores são aparelhos de segurança eletromecânicos usados para seccionamento de circuitos (MAMEDE FILHO, 2013). Nas subestações o disjuntor usado será de média tensão com proteção mínima de sobrecorrente. O disjuntor deve possuir acionamento automático na abertura, com seu comando de abertura, trip, dado por relé microprocessado, sendo eles a vácuo ou com qualquer líquido isolante não inflamável, com as características mínimas descritas na Norma ND 5.3 da CEMIG.

O relé de proteção microprocessado é responsável pelo seccionamento do disjuntor de média tensão. Ele deve possuir as funções 50 e 51 e deve atuar em alterações de fase de neutro. Este tipo de proteção é considerada secundária, uma vez que a mesma não tem 
contato direto com a tensão e corrente nominal da subestação, sendo então necessário o uso de TCs e TP abaixadores que enviam sinais de corrente e tensão em valores que o relé é capaz de processar (MAMEDE FILHO; MAMEDE, 2011).

Os sinais de tensão e corrente dos TP e TCs devem ser comparados com variáveis calculadas nos projetos e apresentadas junto com o coordenograma de proteção e nele inseridas. Estes sinais são responsáveis pelo trip ou não do disjuntor. Demais informações sobre os parâmetros calculados e funções mínimas que o relé deve possuir estão descritas na Norma da CEMIG ND 5.3.

Outro equipamento importante é a chave seccionadora tripolar com fusível, entre as seções de medição, proteção, transformação e saída deverá existir um sistema de seccionamento com função de isolar cada seção. O equipamento responsável por esse seccionamento é a chave seccionadora tripolar. A chave deve possuir abertura conjunta em todas as fases, possuir proteção contra sobrecorrente por meio de fusíveis de rápida atuação, além disto, deve possuir atuação manual com acionamento sobre alavanca. $\mathrm{O}$ acionamento manual é usado para a desenergização da subestação para limpeza e conservação ou quaisquer que seja o fator de segurança.

A fim de evitar sobrecarga, todas as partes metálicas dos equipamentos devem ser aterradas, o aterramento deve percorrer toda a subestação. O número de hastes e a bitola do aterramento são proporcionais à demanda de potência da subestação, porém, independente da potência a medição ôhmica em qualquer uma das hastes deve ser menor que $10 \Omega$, em qualquer época do ano. Além disto, o cabo de neutro da concessionária deve ser conectado ao aterramento da subestação.

\subsection{Transformadores}

Transformadores são dispositivos físicos de indução eletromagnética, capazes de aumentar ou reduzir valores de tensão e corrente (JORDÃO, 2002). Podem ser a óleo isolante ou a seco usando epóxi como isolante. O equipamento deve possuir laudo técnico e estar consonante com suas normas de uso expedidas pela ABNT e demais órgãos reguladores.

Em subestações da CEMIG, o primário do transformador deve ser em delta e seu secundário em estrela a quatro fios, com seu neutro conectado ao aterramento da subestação. A instalação do "trafo" deve ser feita em lugares ventilados e que permitam manutenção, remoção e operação. Quando a subestação ocupar parte constituinte da edificação, sua porta de acesso será na parte interna da edificação sendo obrigatório o uso de transformadores a 
seco e encapsulados por epóxi, mesmo se a subestação constar com paredes de alvenaria e portas corta-fogo (CEMIG, 2013).

\subsection{Equipamentos de Ligação}

Existem materiais responsáveis pela condução de corrente entre os setores de medição, proteção e transformação que são os barramentos, podendo ser de cobre ou alumínio, e seus conectores. Seu dimensionamento, conforme corrente por ele transportada, e distância entre fases para subestações da CEMIG, estão contidas na norma ND 5.3.

Além disto, existem as buchas de passagem que fazem a transição dos condutores de um setor ao outro. AS buchas possuem condutor encapsulado por porcelana e fixados por chapas nas paredes de separação.

\section{RESULTADOS E DISCUSSÕES}

Neste capítulo será apresentado levantamento de carga, dimensionamento dos equipamentos de medição e proteção, malha de aterramento e, por fim, planta baixa em corte e diagrama unifilar para melhor visualização da subestação.

\subsection{Levantamento de Carga}

Inicialmente, para o cálculo da demanda do consumidor, foi realizado um levantamento de carga instalada no local. Basicamente a carga é constituída por lâmpadas fluorescentes, lâmpadas de vapor de mercúrio e vários motores trifásicos que variam entre si e em relação a suas tensões de acionamento, quantidade e potência. Assim após levantamento foi confeccionada tabela de cargas abaixo (Tabela 2). 
Tabela 2 - Levantamento de Carga Fábrica de Refrigerantes Mantiqueira

\begin{tabular}{|c|c|c|c|c|c|c|c|}
\hline \multicolumn{8}{|c|}{ Relação de Cargas } \\
\hline Item & Descrição & Fases & Tensão & Quantidade & $\mathrm{CV}$ & $\mathrm{kVA}$ total & FP \\
\hline 1 & Lâmpada fluorescente $32 \mathrm{~W}$ & 1 & 220 & 165 & - & 5,271 & 0,85 \\
\hline 2 & Lâmpada V.M. 250W & 1 & 220 & 25 & - & 7,353 & 0,85 \\
\hline 3 & Macaco eletromecânico & 4 & 380 & 4 & 0,5 & 1,72 & 0,85 \\
\hline 4 & Motor trifásico tipo 2 & 3 & 220 & 19 & 1 & 15,77 & 0,85 \\
\hline 5 & Motor trifásico tipo 3 & 3 & 220 & 2 & 1,5 & 2,44 & 0,85 \\
\hline 6 & Motor trifásico tipo 4 & 3 & 220 & 15 & 2 & 21,60 & 0,85 \\
\hline 7 & Motor trifásico tipo 5 & 3 & 220 & 28 & 3 & 61,04 & 0,85 \\
\hline 8 & Motor trifásico tipo 6 & 3 & 220 & 2 & 4 & 5,96 & 0,85 \\
\hline 9 & Motor trifásico tipo 7 & 3 & 220 & 10 & 5 & 33,70 & 0,85 \\
\hline 10 & Motor trifásico tipo 8 & 3 & 220 & 10 & 10 & 64,60 & 0,85 \\
\hline 11 & Motor trifásico tipo 9 & 3 & 220 & 6 & 20 & 74,40 & 0,85 \\
\hline 12 & Motor trifásico tipo 10 & 3 & 220 & 5 & 25 & 74,00 & 0,86 \\
\hline 13 & Motor trifásico tipo 11 & 3 & 220 & 1 & 50 & 49,27 & 0,86 \\
\hline 14 & Motor trifásico tipo 12 & 3 & 220 & 2 & 75 & 140,96 & 0,86 \\
\hline & Total Cargas [Kva] & & & & & 558,08 & \\
\hline
\end{tabular}

Fonte: Acervo Pessoal (2017)

A carga total instalada é de 558,08kVA, conforme ilustrado em Tabela 2, o consumidor irá ser atendido por uma subestação tipo 2, Medição, Proteção e Transformação padrão CEMIG. Entrada e saída da subestação serão subterrânea, transformador de 1MVA, 13800/220/127. O transformador usado é de carga superior à demanda devido à expectativa de expansão futura da fábrica. A carga a ser contratada inicialmente será de $400 \mathrm{~kW}$, sendo aumentada após expansão futura para 1MVA, prevista para o segundo semestre de 2018.

\subsection{Medição do Consumo de Energia Elétrica}

O setor de medição será composto por TCs de sinal de 40/5 com fator de transformação de 1,5; TPs de sinal de fator de transformação de 70/1, ambos serão instalados em mesa suporte e seus condutores de sinal direcionados a caixa de medição CM-4 por eletrodutos galvanizados de 1.1/4 polegadas. No interior da CM-4 são instalados os medidores de consumo e, por fim, a caixa é lacrada, ilustrado nas Figuras 2 e 3. 


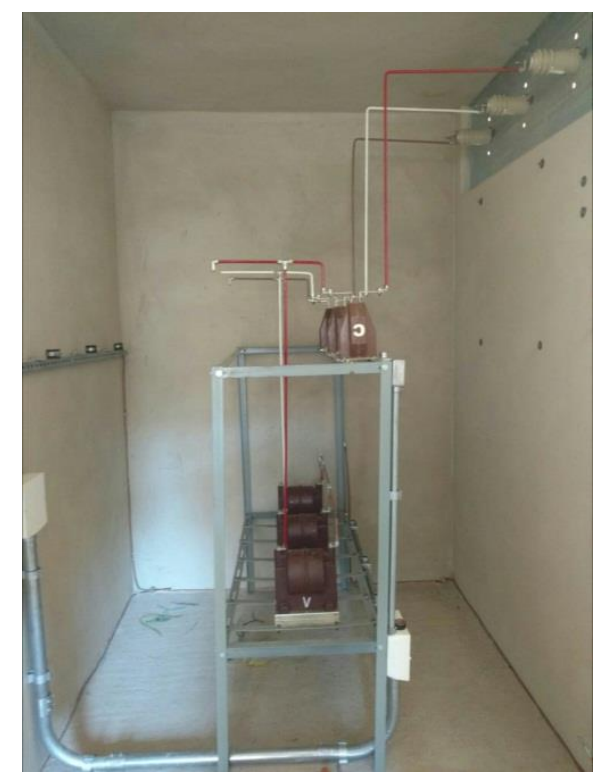

Figura 2 - Execução de baia de medição de Subestação Fonte: Acervo Pessoal (2017)

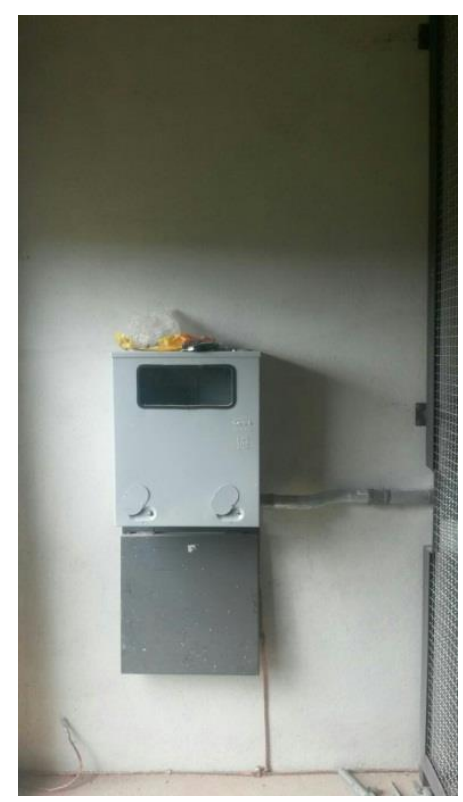

Figura 3 - Instalação de caixa de medição CM-4 Fonte: Acervo Pessoal (2017)

\subsection{Setor de Proteção}

No setor de proteção, está o disjuntor em média tensão a óleo isolante, três TCs de sinal de proteção de 100/5 e um TP de 13800/220/127 V usado para a alimentação do relé microprocessado que fica fora da baia de proteção, ilustrado na Figura 4. 


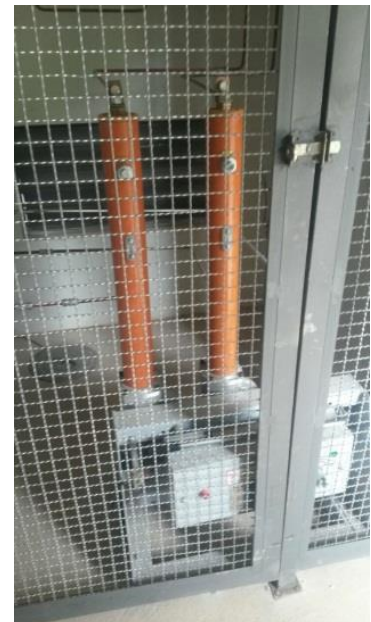

Figura 4 - Execução de setor de proteção.

Fonte: Acervo Pessoal (2017)

Todos os cabos de sinal são direcionados para o relé por eletroduto galvanizado de 1 pol. O relé utilizado é o Pextron 7104, ilustrado na Figura 5, instalado em caixa basculante contendo as funções $50,50 \mathrm{~N}, 51$ e $51 \mathrm{~N}$; controlando qualquer alteração de corrente instantânea ou temporizada. Todos os parâmetros nele inseridos estão calculados no projeto aprovado pela CEMIG e apresentados em Tabela 3.

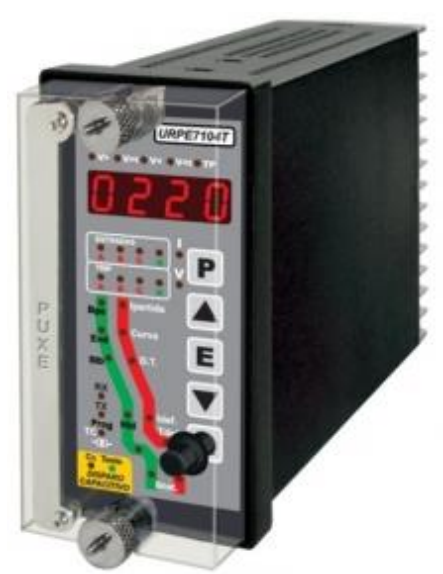

Figura 5 - Relé de proteção Pextron® 7104T

Fonte: Pextron (2016) 
Tabela 3 - Dados de Parametrização do Relé

\begin{tabular}{|c|c|c|}
\hline \multicolumn{3}{|c|}{ Parâmetros e Ajustes } \\
\hline Parâmetro & Descrição do parâmetro & Faixa \\
\hline \multicolumn{3}{|c|}{ Fase } \\
\hline TC & Relação do transformador de corrente TC & 20 \\
\hline I partida & Corrente de partida $(\mathrm{A})$ & 19,12 \\
\hline Curva & Tipo de curva de atuação de fase & EI \\
\hline D.T. & Ajuste do dial de tempo para fase (s) & 0,50 \\
\hline I def. & Corrente de partida tempo definido de fase (A) & Max. \\
\hline T def. & Tempo da unidade definido de fase (s) & Max. \\
\hline I inst. & Corrente instantânea de fase (A) & 351,79 \\
\hline \multicolumn{3}{|c|}{ Neutro } \\
\hline I partida & Corrente de partida (A) & 6,31 \\
\hline Curva & Tipo de curva de atuação para neutro & EI \\
\hline D.T. & Ajuste do dial de tempo para neutro (s) & 0,50 \\
\hline I def. & Corrente de partida tempo definido de neutro (A) & Max. \\
\hline T def. & Tempo da unidade definido de neutro (s) & Max. \\
\hline I inst. & Corrente instantânea de neutro (A) & 116,10 \\
\hline
\end{tabular}

Fonte: Acervo Pessoal (2017)

O circuito de comando de abertura do disjuntor através de comando de relé microprocessado é ilustrado na Figura 6.

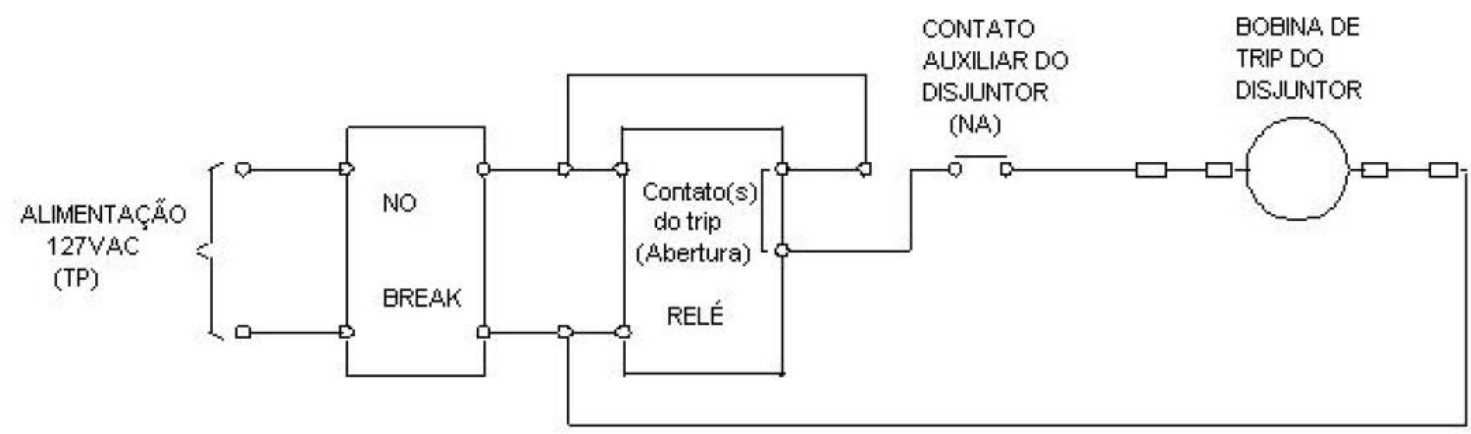

Figura 6 - Circuito de comando de abertura do disjuntor através de comando de relé microprocessado. Fonte: CEMIG (2013)

\subsection{Aterramento}

Em uma instalação elétrica, o aterramento elétrico é realizado mediante um fio ligado diretamente à terra. $\mathrm{O}$ fio é conectado a corpos de metais que estão cravados na terra. A finalidade do aterramento é desviar o excesso de corrente do equipamento elétrico, evitando sobrecarga. Geralmente, o aterramento é feito em malha e conectados a corpos de metais chamados hastes ou eletrodos (NBR 14039, 2005). 
A normativa da CEMIG ND 5.3, estabelece que para cargas maiores que 500kVA o número mínimo de hastes que deve haver na malha de aterramento são 12 hastes. A malha de aterramento da subestação está ilustrada na Figura 7.

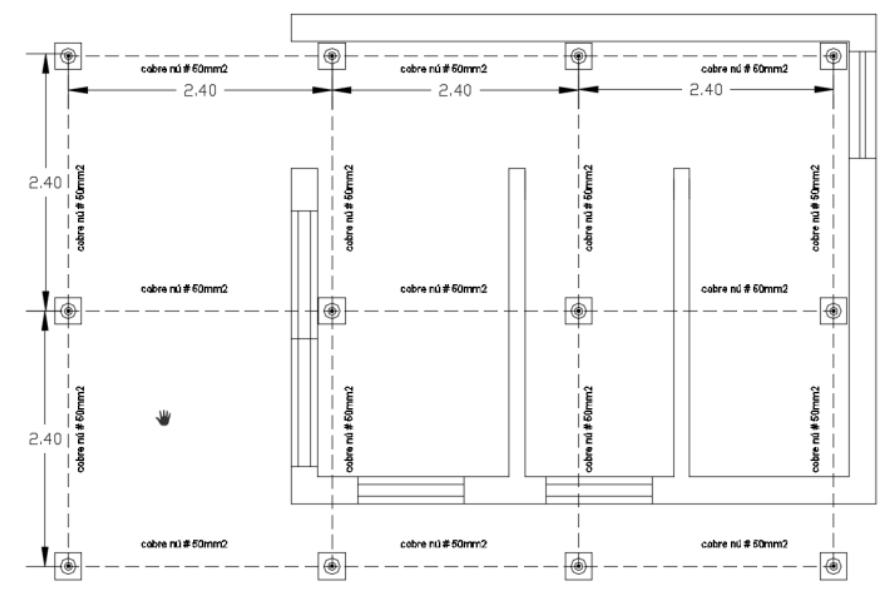

Figura 7 - Malha de aterramento subestação tipo 2 Fábrica de Refrigerantes Mantiqueira Fonte: Acervo Pessoal (2017)

Primeiramente nota-se que a malha de aterramento ultrapassou os limites da subestação, respeitando os parâmetros mínimos da norma. A malha de aterramento possui resistência ôhmica menor ou igual a $10 \Omega$ em todos os pontos vistoriados pela CEMIG. Todas as cavas de aterramento possuem $25 \mathrm{~cm}$ de lado e $50 \mathrm{~cm}$ de profundidade e após fechado o aterramento são preenchidas com massa de cimento e brita, além de serem tapadas com tampas de ferro. Para as hastes de aterramento são usadas cantoneiras de $25 \mathrm{~mm}$ de lado e $5 \mathrm{~mm}$ de espessura e fincadas a 2,40m de profundidade.

O neutro da instalação, da Subestação e da CEMIG também é conectado ao aterramento. Todas as partes metálicas de materiais, equipamentos, portas, etc. são aterradas, porém para estes materiais o condutor de aterramento possui a seção de $25 \mathrm{~mm}^{2}$.

\subsection{Informações adicionais}

Os condutores de corrente da subestação são de cobre de 3/8 de polegada, na transição dos setores são usadas buchas de passagem de porcelana fixadas em placas metálicas. Além disto, na entrada dos setores de proteção e transformação existem chaves seccionadoras tripolares de $15 \mathrm{kV}$, com abertura sobre carga e com fusível de rápida atuação de 200A. Abaixo, vê-se a planta baixa da montagem da subestação e corte longitudinal de modo a melhorar a visualização (Figuras 8 e 9). 


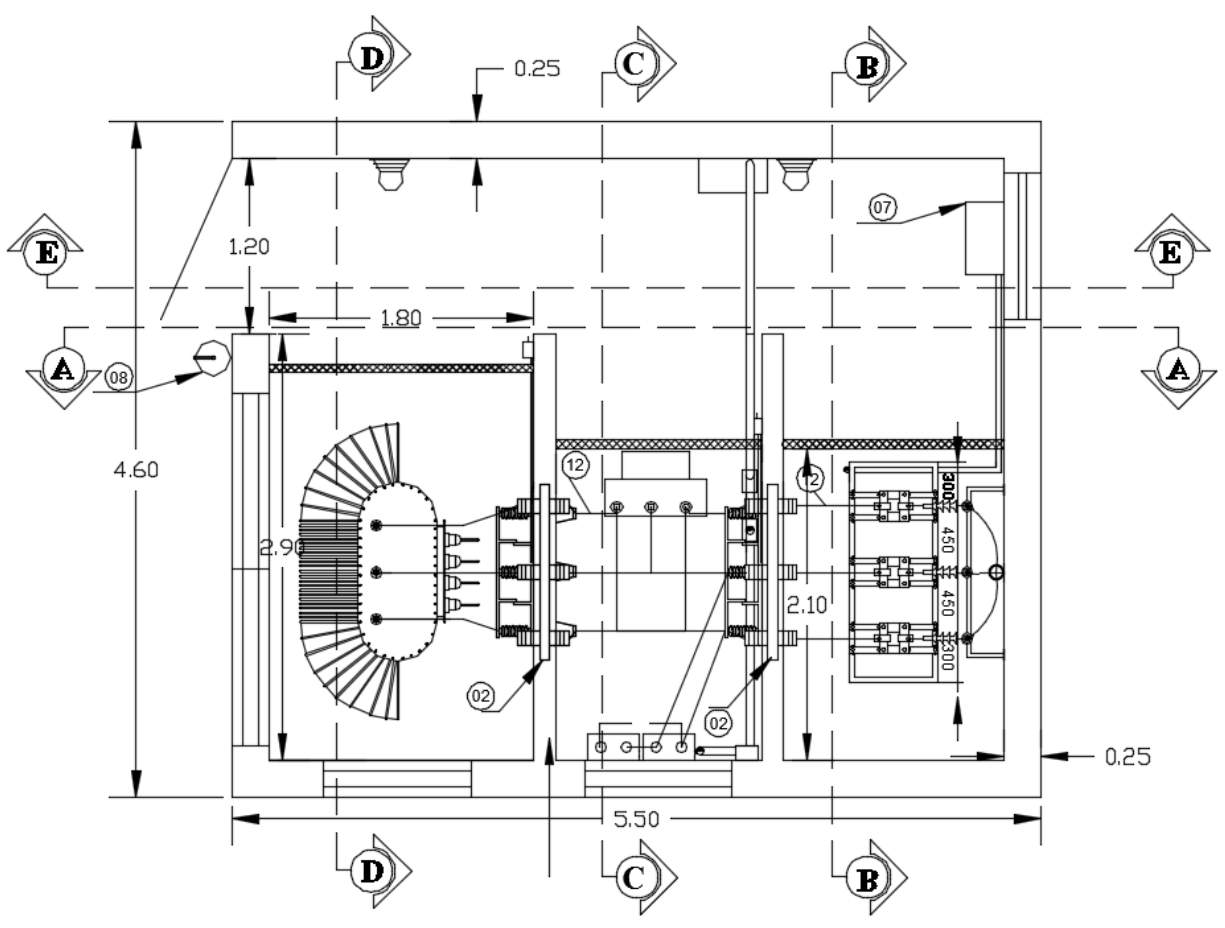

Figura 8 - Planta baixa Subestação Fábrica de Refrigerantes Mantiqueira Fonte: Acervo Pessoal (2017)

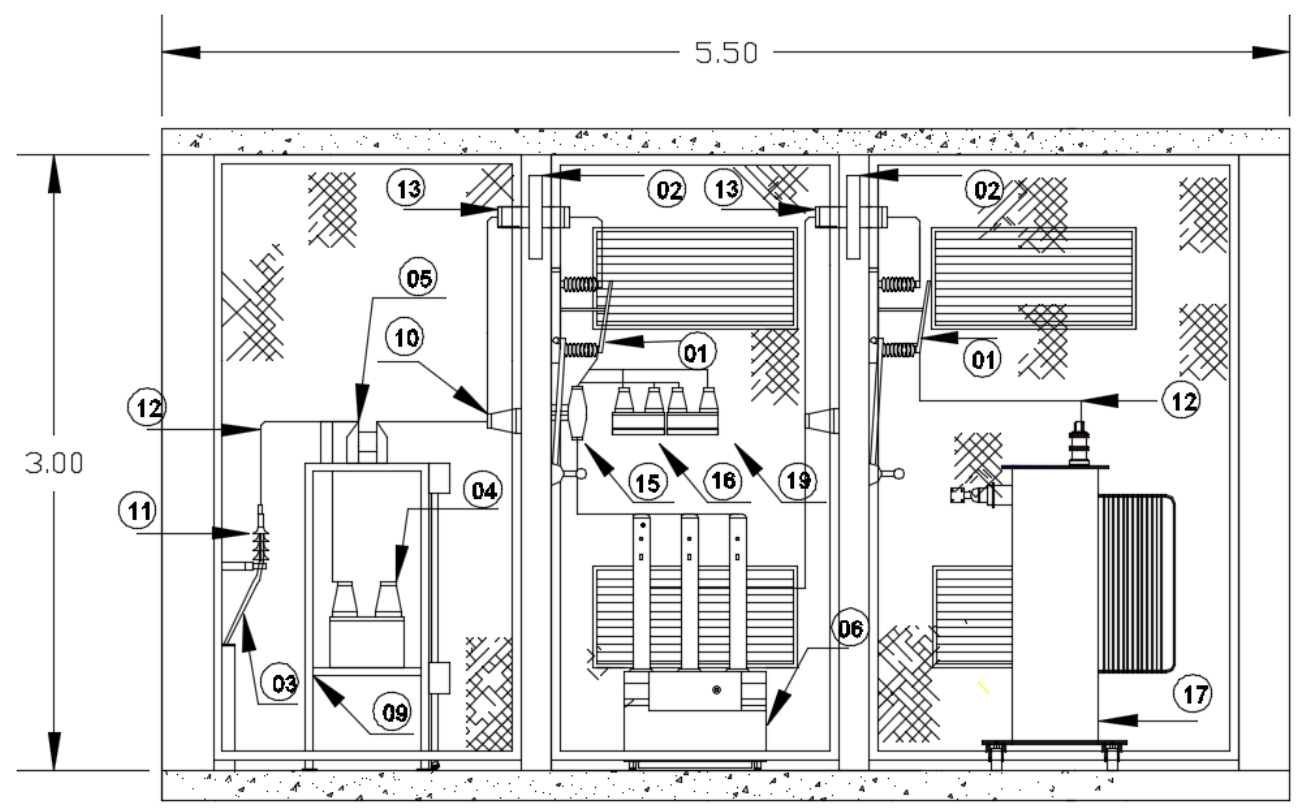

Figura 9 - Corte de planta longitudinal.

Fonte: Acervo Pessoal (2017)

Fica então definido por diagrama unifilar, ilustrado na Figura 10, a construção e as ligações de cada setor da subestação apresentada. 


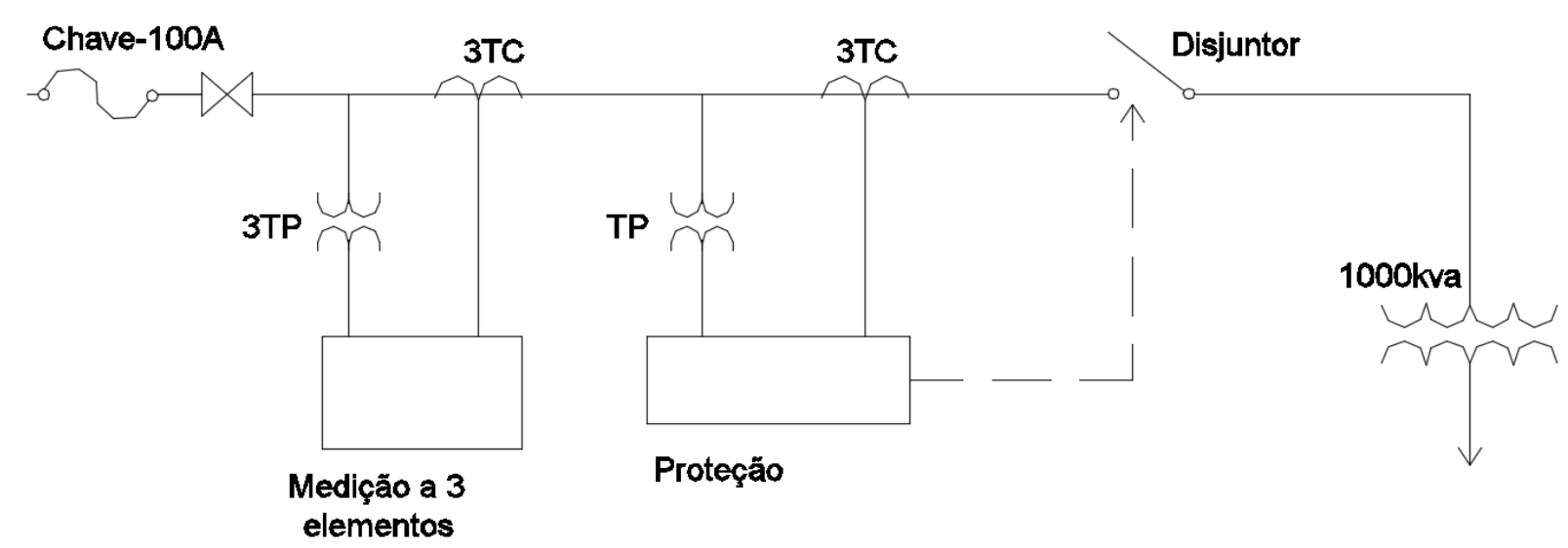

Figura 10 - Diagrama Unifilar Fábrica de Refrigerantes Mantiqueira

Fonte: Acervo Pessoal (2017)

\section{CONCLUSÃO}

\subsection{Considerações Finais}

O ramo industrial de engenharia elétrica é muito vasto, principalmente em média tensão. Destaca-se também que a subestação de energia em média tensão é um campo que requer muito estudo e responsabilidade do responsável técnico, incluindo requisitos associados com perícia e acurácia aos técnicos de montagem.

O objetivo deste trabalho era o desenvolvimento, desde os projetos até a execução de uma subestação, executando com o menor custo e exercendo suas funções de maneira adequadas. Inicialmente foi feita pesquisa bibliográfica necessária para conhecimento de normas e resoluções para a projeção das subestações. Toda a execução da subestação, desde a aquisição dos materiais a instalação dos mesmos, foi acompanhada pelo autor deste trabalho.

O projeto, o desenvolvimento e, por fim, a construção da subestação apresentados neste artigo, foram aprovados e elogiados em vistoria feita pela CEMIG. Importante citar que as empresas responsáveis pela obra possuem grande experiência neste mercado ainda não muito disseminado pelo Brasil, principalmente em Minas Gerais.

\subsection{Trabalhos Futuros}

O conhecimento e experiências adquiridos não são válidos somente para compra de energia elétrica, mas, também, para venda de energia gerada por meios incentivados e 
renováveis como biomassa, solar, entre outras que, necessitam da montagem de subestações elevadoras de tensão para inserir a energia gerada na rede de transmissão.

Ressalta-se que, após a conclusão dos trabalhos da subestação de energia elétrica e a compra de energia, há ainda trabalhos a serem realizados. Um exemplo é o ajuste do fator de potência do consumidor, calculado pelo memorial de massa de consumo de energia elétrica e corrigido por bancos de capacitores. Outro exemplo é o comando de partida de motores e quaisquer manutenções elétricas industriais necessárias para o contínuo funcionamento da produção.

\title{
DEVELOPMENT OF MEDIUM VOLTAGE SUBSTATION BOOTH
}

\begin{abstract}
Nowadays, electrical energy is indispensable for human life due to continuous technological evolution, noticeable, for instance, on the increase of industrial automation systems, interconnection of knowledge networks, among others. Industrial units and large commercial conglomerates demand a large proportion of the energy generated in our country. Thus, they often choose to purchase electrical energy in medium/high voltage. Such choice is worth for its low cost (when compared to low voltage supply). The monetary difference is justified because the medium/high voltage power flow requires less electrical current, reducing the cross-section of the transmission conductor, and, thus, reducing the structure of the transmission towers for cable supporting. This economy is passed on to the customers in forms of lower energy tariff values. For the supply of electrical energy in medium voltage, it is necessary that the consumer unit complies with certain requirements proposed by the energy concessionaire, in the case of Minas Gerais, by the Companhia de Energia de Minas Gerais (CEMIG®). Among these requirements, there is the construction of one of the seven types of medium voltage electric substation (CEMIG, 2013). The different models come from differences associated to size, number of bays, installed power and energy input, among others. Thus, this paper aims to present the construction of a type 2 power substation Measurement, Protection with or without Transformation (CEMIG, 2013). Presenting a case study of the mentioned work in the city of Itamonte-MG, in Mantiqueira Soft drinks factory (Fábrica de Refrigerantes Mantiqueira).
\end{abstract}

Keywords: Electrical Energy Supply, Substation, Medium Voltage, CEMIG®.

\section{REFERÊNCIAS}

AGÊNCIA NACIONAL DE ENERGIA ELÉTRICA. Módulos construtivos de subestações de distribuição: catálogo referencial de módulos padrões de construção de subestações, linhas e redes de distribuição de energia elétrica. 2010. Disponível em: <http://www2. aneel.gov.br/aplicacoes/audiencia/arquivo/2009/052/documento/anexo_iii_nt_304__subestacao_de_distribuicao.pdf >. Acesso em: 24 mar. 2017. 
Banco de informação de geração BIG. 2017. Disponível em: <http://www2. aneel.gov.br/aplicacoes/capacidadebrasil/capacidadebrasil.cfm>. Acesso em: 24 mar. 2017.

Resolução $\mathbf{n}^{\circ}$ 414, de 09 de setembro de 2010. Estabelece as condições gerais de fornecimento de energia elétrica de forma atualizada e consolidada. 2010. Disponível em: <http://www.aneel.gov.br/documents/656877/14486448/bren2010414.pdf/3bd33297-26f94ddf-94c3-f01d76d6f14a?version=1.0>. Acesso em: 20 mar. 2017.

ASSOCIAÇÃO BRASILEIRA DE NORMAS TÉCNICAS. NBR 14039: instalações elétricas em média tensão. Rio de Janeiro, 2005.

NBR 5410: instalações elétricas em baixa tensão. Rio de Janeiro, 2008.

BARROS, B. F.; GEDRA R. L. Cabine primária: subestação de alta tensão de consumidor. 2. ed. São Paulo: Ed. Érica, 2013.

CELESC. O mercado de energia. c2011. Disponível em: <http://novoportal.celesc.com.br/ portal/index.php/celesc-geracao/comercializacao/o-mercado-de-energia>. Acesso em: 10 jan. 2016.

COMERC ENERGIA. O mercado livre de energia. 2016. Disponível em: <http://www. comerc.com.br/comerc/o_mercado_livre_de_energia.asp>. Acesso em: 10 jan. 2016.

COMPANHIA ENERGÉTICA DE MINAS GERAIS. ND 5.2: fornecimento de energia elétrica em tensão secundária rede de distribuição aérea edificações coletivas. Belo Horizonte, 2015.

ND 5.3: fornecimento de energia elétrica em média tensão rede de distribuição aérea ou subterrânea. Belo Horizonte, 2013.

EMPRESA DE PESQUISA ENERGÉTICA. Consumo anual de energia elétrica por classe (nacional): 1995-2014. 2014. Disponível em: <http://www.epe.gov.br/mercado/Paginas/ Consumonacionaldeenergiael\%C3\%A9tricaporclasse\%E2\%80\%931995-2009.aspx >. Acesso em: 24 mar. 2017.

JORDÃO, R. B. Transformadores. São Paulo: Blucher, 2002.

KAGAN, N.; OLIVEIRA, C. C. B.; ROBBA, E. J. Introdução aos sistemas de distribuição de energia elétrica. São Paulo: Blucher, 2005.

MAMEDE FILHO, J. Manual de equipamentos elétricos. 4. ed. São Paulo: LTC, 2013.

MAMEDE FILHO, J; MAMEDE, D. R. Proteção de sistemas elétricos de potência. São Paulo: LTC, 2011.

NORMA Regulamentadora 10 - NR 10: segurança em instalações e serviços em eletricidade. 2004. Disponível em: <http://www.guiatrabalhista.com.br/legislacao/nr/nr10.htm>. Acesso em: 5 set. 2016. 
PEXTRON. URPE 7104. Disponível em: <http://www.pextron.com.br/produtos/reles-deprotecao-multifuncao/urpe-7104-sobrecorrente-trifasico.html $>$. Acesso em: 2 jul. 2015.

Recebido em: 23/05/2017

Aprovado em: 30/05/2017

Publicado em: 28/06/2017 